\title{
The $\alpha$-element abundances in the most oxygen-poor planetary nebula PNG 135.9+55.9
}

\author{
M. G. Richer ${ }^{1, \star}$, G. Tovmassian ${ }^{1}$, G. Stasińska ${ }^{2}$, R. F. Jameson ${ }^{3, \star \star}$, P. D. Dobbie ${ }^{3, \star \star}$, C. Veillet ${ }^{4, \star \star \star}$, \\ C. Gutierrez ${ }^{5, \dagger}$, and F. Prada ${ }^{6, \dagger}$ \\ 1 Observatorio Astronómico Nacional, Instituto de Astronomía, UNAM, PO Box 439027, San Diego, CA 92143-9027, USA \\ e-mail: \{richer, gag\}@astrosen.unam.mx \\ 2 LUTH, Observatoire de Meudon, 5 place Jules Janssen, 92195 Meudon Cedex, France \\ e-mail: grazyna.stasinska@obspm.fr \\ 3 University of Leicester, University Road, Leicester LE1 7RH, UK \\ e-mail: $\{r f j$, pdd\}@star.le.ac.uk \\ ${ }^{4}$ Canada-France-Hawaii Telescope Corp., PO Box 1597, Kamuela, HI 96743, USA \\ e-mail: veillet@cfht.hawaii.edu \\ 5 Instituto de Astrofísica de Canarias, C/Vía Láctea s/n, 38200, La Laguna, Tenerife, Spain \\ e-mail: cgc@ll.iac.es \\ ${ }^{6}$ Centro Astronómico Hispano Alemán, C/Jesús Durbán Remón 2-2, 04004, Almería, Spain \\ e-mail:prada@caha.es
}

Received 26 June 2002 / Accepted 9 September 2002

\begin{abstract}
We present extensive new spectroscopy and imaging of PNG 135.9+55.9. We use these data as constraints to photoionization models to derive limits on the oxygen abundance. We find that PNG 135.9+55.9 has an oxygen abundance less than $1 / 50$ of the solar value. Our models favour a value of $12+\log \mathrm{O} / \mathrm{H}$ between 5.8 and $6.5 \mathrm{dex}$, confirming that PNG 135.9+55.9 is the most oxygen-poor planetary nebula known (Tovmassian et al. 2001). We also derive $\mathrm{Ne} / \mathrm{O}=0.5 \pm 0.3, \mathrm{~S} / \mathrm{O}<0.094$, and $\mathrm{Ar} / \mathrm{O}<0.23$. Although the value of $\mathrm{Ne} / \mathrm{O}$ is nominally high, it need not imply that the progenitor of PNG 135.9+55.9 converted any of its initial oxygen abundance to neon. The helium abundance appears to be very low, $\mathrm{He} / \mathrm{H} \sim 0.08$, but a precise determination will require a much more detailed study. We find that $\mathrm{H} \alpha / \mathrm{H} \beta$ is lower than expected and perhaps variable, a finding for which we have no clear explanation.
\end{abstract}

Key words. planetary nebulae: individual: PNG 135.9+55.9

\section{Introduction}

Recently, SBS 1150+599A has been recognized as a planetary nebula in the Galactic halo by Tovmassian et al. (2001) and renamed PNG 135.9+55.9. The spectra then available for this

Send offprint requests to: $\mathrm{M}$. Richer,

e-mail: richer@astrosen.unam.mx

* Visiting Astronomer, Observatorio Astrónomico Nacional in San Pedro Mártir, Mexico.

$\star \star$ Visiting Astronomer, William Herschel Telescope, operated by the Isaac Newton Group (ING) on behalf of the UK Particle Physics and Astronomy Research Council (PPARC), the Nederlanse Organisatie voor Wetenschappelijk Onderzoek (NWO), and the Instituto de Astrofsica de Canarias (IAC).

$\star \star \star$ Visiting Astronomer, Canada-France-Hawaii Telescope operated by the National Research Council of Canada, the Centre National de la Recherche Scientifique de France and the University of Hawai'i.

$\dagger$ Visiting Astronomer, Nordic Optical Telescope, operated by the ING on behalf of the PPARC, NWO, and IAC. object were quite unusual for a planetary nebula, presenting only the Balmer lines of hydrogen, He II $\lambda \lambda 4686,5411$, and very weak [O III] $\lambda 5007(\sim 4 \%$ of $\mathrm{H} \beta)$. A photoionization model analysis showed that such a spectrum implies a strongly density bounded and extremely oxygen-poor nebula ionized by a very hot star. The oxygen abundance was estimated to be less than $1 / 50$ of the solar value, and probably between $1 / 100$ and $1 / 500$ of solar assuming canonical properties for the central star, making of PNG 135.9+55.9 by far the most oxygen-poor planetary nebula known, with an oxygen abundance similar to the lowest measured to date in stars (Boesgaard et al. 1999; Howard et al. 1997).

In this paper, we report detailed follow-up observations, aimed at providing more stringent constraints on the nature of this exceptional object. Section 2 presents the new spectroscopic data, while Sect. 3 deals with narrow-band imaging. In Sect. 4, we present an updated photoionization model analysis, taking full advantage of the constraints provided by our new 
Table 1. Log of the spectroscopic observations.

\begin{tabular}{|c|c|c|c|c|c|c|}
\hline & SPM1 & SPM2 & CFHT & WHT & $\mathrm{SPM}^{a}$ & SPM4 \\
\hline date & 22 Jan. 2001 & 23-24 Jan. 2001 & 3-4 Mar. 2001 & 11 May 2001 & 5 Mar. 2002 & 8 Apr. 2002 \\
\hline CCD & Thomson & Thomson & EEV & Tektronix & SITe & SITe \\
\hline format & $2048 \times 2048$ & $2048 \times 2048$ & $2048 \times 4500$ & $1024 \times 1024$ & $1024 \times 1024$ & $1024 \times 1024$ \\
\hline pixel size ${ }^{b}$ & $14 \mu \mathrm{m}, 0^{\prime} .61$ & $14 \mu \mathrm{m}, 0^{\prime} 61$ & $13.5 \mu \mathrm{m}, 0 . .283$ & $24 \mu \mathrm{m}, 0.36$ & $24 \mu \mathrm{m}, 1^{\prime \prime} .05$ & $24 \mu \mathrm{m}, 1^{\prime \prime} 05$ \\
\hline gain $\left(\mathrm{e}^{-} / \mathrm{ADU}\right)$ & 0.5 & 0.5 & 1.8 & 1.4 & 1.3 & 1.3 \\
\hline read noise $\left(\mathrm{e}^{-}\right)$ & 4.8 & 4.8 & 3.1 & 4.6 & 8 & 8 \\
\hline spectrograph & $\mathrm{B} \& \mathrm{C}$ & $\mathrm{B} \& \mathrm{C}$ & MOS & ISIS & $\mathrm{B} \& \mathrm{C}$ & $\mathrm{B} \& \mathrm{C}$ \\
\hline grating $^{c}$ & $300 / 4550 \AA$ & $600 / 4550 \AA$ & B400/5186 A & $\mathrm{R} 158 \mathrm{R} / 6500 \AA$ & $300 / 4550 \AA$ & $400 / 5150 \AA$ \\
\hline slit width & 3.' 8 & 3.' 8 & $5^{\prime \prime}$ & $1^{\prime \prime}$ & 2.3 & $2 . .3$ \\
\hline spectral resolution $^{d}$ & $12.7 \AA$ & $5.6 \AA$ & $23.0 \AA$ & $7.8 \AA$ & $8.1 \AA$ & $5.8 \AA$ \\
\hline wavelength interval & $3700-6700 \AA$ & $4745-7200 \AA$ & $3400-8000 \AA$ & $6820-9740 \AA$ & $3600-7100 \AA$ & $3660-6760 \AA$ \\
\hline arc lamp & $\mathrm{HeAr}$ & $\mathrm{HeAr}$ & $\mathrm{HgNeAr}$ & $\mathrm{CuNe}+\mathrm{CuAr}$ & $\mathrm{HeAr}$ & $\mathrm{HeAr}$ \\
\hline \multirow[t]{2}{*}{ standard stars } & HD 93521 & G191B2B & Feige 66 & $\mathrm{~Hz} 44$ & $\mathrm{BD}+33^{\circ} 2642$ & HD 93521 \\
\hline & $\mathrm{BD}+33^{\circ} 2642$ & HD 93521 & & & & $\mathrm{BD}+33^{\circ} 2642$ \\
\hline total exp. time & $4500 \mathrm{~s}$ & $7800 \mathrm{~s}$ & $7200 \mathrm{~s}$ & $3600 \mathrm{~s}$ & $5400 \mathrm{~s}$ & $12600 \mathrm{~s}$ \\
\hline number of spectra & 3 & 4 & 4 & 3 & 3 & 7 \\
\hline
\end{tabular}

${ }^{a}$ These observations were obtained through clouds.

${ }^{b}$ Both the physical pixel size and the angle subtended on the sky are given.

${ }^{c}$ The gratings are described by their ruling (lines/mm) and effective blaze wavelength. For the grism used at CFHT, the ruling and zero deviation wavelength are given. All of the observations were obtained in first order.

${ }^{d}$ This is the spectral resolution measured at $\mathrm{H} \alpha$, defined as the $F W H M$ of the $\mathrm{H} \alpha$ line, except for the WHT spectrum, where this is the $F W H M$ of P9ג9229.

observational data. This leads to a limit on the oxygen abundance which is now independent of any assumption about the evolutionary status of the central star. In Sect. 5, we estimate the abundances of the other elements. Section 6 presents a brief concluding discussion.

\section{Spectroscopy}

\subsection{Observations}

Table 1 presents a summary of our new spectroscopic observations of PNG 135.9+55.9. This table includes the dates of the observations, the instrumental configuration, and the flux and wavelength standards that were used. The only observing run that suffered from non-photometric conditions was that of 5 Mar. 2002, when significant cloud cover affected observations of both PNG 135.9+55.9 and the standard star.

The spectroscopy from the Observatorio Astronómico Nacional in San Pedro Mártir, Baja California, Mexico (SPM) was obtained using the Boller \& Chivens spectrograph (B\&C) and three different gratings during four observing runs. For the 2001 observations, a rather wide slit (3".8) was used to better measure the total fluxes, while, for the 2002 observations, a narrower slit was used to obtain higher spectral resolution and better sensitivity to fainter lines. The standard stars were observed with an even wider slit $\left(9^{\prime \prime}\right)$. In all cases, the slit was oriented east-west on the sky. Spectra of the illuminated dome wall were obtained to serve as flat field images. Bias images were obtained at the beginning and end of the night.
The spectroscopy at the Canada-France-Hawaii Telescope (CFHT) was obtained with the Multi-Object Spectrograph (MOS; Le Fèvre et al. 1994). Both the object and the standard star were observed through a 5 " slit. However, the observations of PNG 135.9+55.9 were obtained the night before those of the standard star. Spectra of the internal halogen lamp were obtained to serve as flat field images.

The spectroscopy at the William Herschel Telescope (WHT) was obtained using the red arm of the ISIS spectrograph. The object was observed through a 1 " slit, while the standard stars were observed with a $10^{\prime \prime}$ slit. For these observations, the slit was oriented at the parallactic angle. Spectra of the internal lamp were obtained to serve as flat field images while spectra of the sky were used to correct for the slit illumination.

All of the spectroscopy was reduced using the Image Reduction and Analysis Facility (IRAF) ${ }^{1}$ software package (specifically the specred package). In all cases, the overscan bias was subtracted from each image. For the SPM data, the overscan-subtracted bias images obtained during the night were combined and subtracted from all of the images. Next, the pixel-to-pixel variations were removed by division of the flat field image. For the WHT data, the slit illumination correction was then applied. The sky emission was subtracted during the extraction of the one dimensional spectra by defining sky

\footnotetext{
${ }^{1}$ IRAF is distributed by the National Optical Astronomical Observatories, which is operated by the Associated Universities for Research in Astronomy, Inc., under contract to the National Science Foundation.
} 
Table 2. Raw line intensities relative to $\mathrm{H} \beta$ for the optical spectra ${ }^{a}$.

\begin{tabular}{|c|c|c|c|c|c|c|}
\hline ion & $\lambda$ & SPM1 & SPM2 & CFHT & SPM $^{b}$ & SPM4 \\
\hline$[\mathrm{Ne} v$ & 3426 & & & $<9.6$ & & \\
\hline$\left[\mathrm{O}_{\text {II }}\right]$ & 3727 & & & $<2.1$ & & \\
\hline$[\mathrm{Ne}$ III] & 3869 & & & $1.04 \pm 0.52$ & & \\
\hline $\mathrm{H}_{\mathrm{I}}$ & 3889 & & & $2.92 \pm 0.73$ & & \\
\hline $\mathrm{H}_{\mathrm{I}}$ & 3970 & $10.0 \pm 2.5$ & & $6.11 \pm 0.64$ & $4.9 \pm 2.9$ & $4.5 \pm 2.1$ \\
\hline $\mathrm{H}_{\mathrm{I}}$ & 4101 & $21.4 \pm 2.8$ & & $20.6 \pm 1.2$ & $19.3 \pm 3.7$ & $21.3 \pm 2.8$ \\
\hline $\mathrm{C}_{\text {II }}$ & 4267 & & & $<1.1$ & & $<3.0$ \\
\hline $\mathrm{H}_{\mathrm{I}}$ & 4340 & $41.0 \pm 1.6$ & & $42.05 \pm 0.98$ & $43.8 \pm 2.4$ & $40.7 \pm 1.4$ \\
\hline [O III] & 4363 & $<1.9$ & & $<0.88$ & & $<1.8$ \\
\hline $\mathrm{He}_{\text {II }}$ & 4686 & $76.1 \pm 2.3$ & & $78.6 \pm 1.5$ & $73.0 \pm 2.1$ & $77.8 \pm 1.3$ \\
\hline$[\mathrm{Ar} \mathrm{IV}]^{c}$ & 4711 & $<2.0$ & & $<1.0$ & & $<1.4$ \\
\hline $\mathrm{H}_{\mathrm{I}}$ & 4861 & $100.0 \pm 2.1$ & $100.00 \pm 0.84$ & $100.0 \pm 1.6$ & $100.0 \pm 1.7$ & $100.00 \pm 0.85$ \\
\hline [O III] & 5007 & $2.7 \pm 1.3$ & $2.17 \pm 0.61$ & $2.87 \pm 0.85$ & $3.8 \pm 1.1$ & $2.31 \pm 0.41$ \\
\hline $\mathrm{He}_{\text {II }}$ & 5412 & $5.68 \pm 0.72$ & $5.47 \pm 0.62$ & $5.23 \pm 0.34$ & $5.64 \pm 0.91$ & $5.53 \pm 0.46$ \\
\hline $\mathrm{He}_{\mathrm{I}}$ & 5876 & $<0.47$ & $<0.69$ & $<0.28$ & $<1.4$ & $<0.62$ \\
\hline $\mathrm{H}_{\mathrm{I}}$ & 6563 & $314.7 \pm 7.6$ & $296.8 \pm 2.4$ & $260.3 \pm 4.4$ & $288.0 \pm 4.1$ & $276.6 \pm 2.0$ \\
\hline$\left[\mathrm{N}_{\text {II }}\right]$ & 6583 & $<3.2$ & $<1.4$ & $<0.45$ & $<1.3$ & $<0.74$ \\
\hline$\left[\mathrm{S}_{\mathrm{II}}\right]$ & 6716 & & & & $<1.3$ & $<0.74$ \\
\hline$\left[\mathrm{S}_{\mathrm{II}}\right]$ & 6731 & & & & $<1.3$ & $<0.74$ \\
\hline$F(\mathrm{H} \beta)^{d}$ & & $1.82 \pm 0.03$ & $1.33 \pm 0.01$ & $2.55 \pm 0.03$ & $0.99 \pm 0.01$ & $1.14 \pm 0.01$ \\
\hline$W(\mathrm{H} \beta)^{e}$ & & $68.4 \pm 1.4$ & $61.4 \pm 0.7$ & $65.3 \pm 1.0$ & $61.5 \pm 1.3$ & $53.5 \pm 0.5$ \\
\hline$W(\mathrm{H} \alpha)^{e}$ & & $646 \pm 58$ & $508 \pm 24$ & $541 \pm 32$ & $494 \pm 10$ & $514 \pm 10$ \\
\hline $\mathrm{H} \alpha$ range $^{f}$ & & $304-318$ & $256-350$ & $258-267$ & $265-324$ & $241-321$ \\
\hline
\end{tabular}

${ }^{a}$ When no uncertainty is given, the value represents a $2 \sigma$ upper limit to the flux in the line.

${ }^{b}$ These observations were obtained through clouds.

${ }^{c}$ These upper limits also apply to [Ar Iv] $\lambda 4740$.

${ }^{d}$ This is the total flux in emission measured at $\mathrm{H} \beta$ in units of $10^{-14} \mathrm{erg} \mathrm{s}^{-1} \mathrm{~cm}^{-2}$.

${ }^{e}$ These are the equivalent widths in $\AA$ of $\mathrm{H} \beta$ and $\mathrm{H} \alpha$ in emission.

${ }^{f}$ This is the total range spanned by the values of $\mathrm{H} \alpha / \mathrm{H} \beta$ among the individual spectra.

regions on both sides of the object spectra and interpolating between them with a straight line. The wavelength calibration was performed using arc lamp spectra obtained at the time of the object observations. Finally, the spectra were calibrated in flux using the observations of the standard stars (Table 1) to determine the instrumental sensitivity function. The individual spectra were calibrated in both wavelength and flux before being summed together.

Table 2 presents the raw line intensities relative to $\mathrm{H} \beta$ measured in the optical spectral region for PNG 135.9+55.9, normalized such that $I(\mathrm{H} \beta)=100$. The line intensities presented in Table 2 are those for the summed spectra from each observing run. The line intensities were measured using the software described by McCall et al. (1985). The uncertainties quoted for each line intensity $(1 \sigma)$ include contributions from the fit to the line itself, from the fit to the reference line, and from the noise in the continuum for both the line and reference line. When only a limit is given, this corresponds to a $2 \sigma$ upper limit to undetected lines.

Table 3 presents the raw fluxes and the intensity ratios relative to $\mathrm{H} \beta$ for the WHT spectrum. The only line definitely detected is P9 $19229 ; \mathrm{P} 12 \lambda 8750$ is detected at only the $2 \sigma$
Table 3. Raw line fluxes for the WHT spectra.

\begin{tabular}{lccc}
\hline \hline ion & $\lambda$ & flux $^{a}$ & $F(\lambda) / F(\mathrm{H} \beta)^{b}$ \\
\hline$\left[\mathrm{Ar}_{\text {III }}\right]$ & 7135 & $<0.45$ & $<0.06$ \\
$\mathrm{H}_{\text {I }}$ & 8750 & $5.7 \pm 2.3$ & $0.74 \pm 0.31$ \\
[ $\left._{\text {III }}\right]$ & 9069 & $<0.59$ & $<0.08$ \\
$\mathrm{H}_{\text {I }}$ & 9229 & $20.3 \pm 3.4$ & $2.64 \pm 0.45$ \\
\hline
\end{tabular}

${ }^{a}$ The fluxes are given in units of $10^{-17} \mathrm{erg} \mathrm{s}^{-1} \mathrm{~cm}^{-2}$. When no uncertainty is given, the value is a $2 \sigma$ upper limit to the flux in the line.

${ }^{b}$ These flux ratios are relative to $F(\mathrm{H} \beta)$ measured for the CFHT spectrum on a scale where $I(\mathrm{H} \beta)=100$.

level. Again, when no uncertainty is given, the value represents a $2 \sigma$ upper limit to the line intensity. These line intensities and limits were measured using IRAF's splot routine. The fluxes represent the fluxes measured directly in the summed WHT spectrum. The intensity ratios relative to $\mathrm{H} \beta$ were computed adopting the $\mathrm{H} \beta$ flux from the CFHT spectrum and correcting the WHT fluxes for the difference in the slit widths used. Based upon the spatial profile of $\mathrm{H} \alpha$ from the 


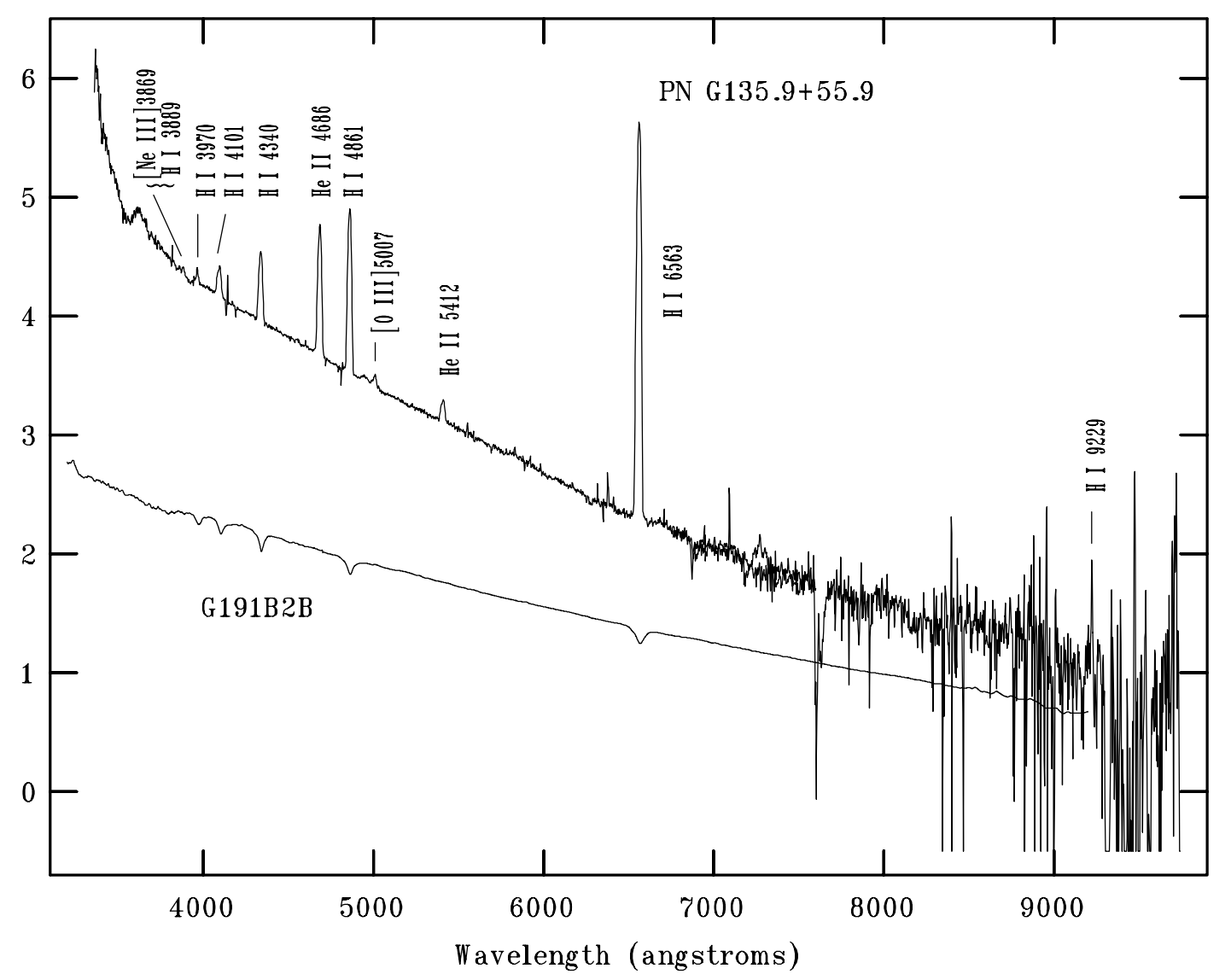

Fig. 1. We compare the spectra of PNG 135.9+55.9 and the standard star G191B2B on an arbitrary magnitude scale. For PNG 135.9+55.9, we plot the CFHT spectrum for $\lambda<7500 \AA$ and the WHT spectrum for $\lambda>6760 \AA$, neither corrected for reddening. The WHT spectrum was normalized to the CFHT spectrum as described in the text. For G191B2B ( $B-V=-0.32 \mathrm{mag}$ ), we plot the Oke (1990) fluxes. As noted by Tovmassian et al. (2001), PNG 135.9+55.9 has a remarkably blue continuum.

CFHT spectrum, a 5" slit intercepts 3.32 times more nebular emission than a $1^{\prime \prime}$ slit. The WHT fluxes were then multiplied by this factor when computing the relative intensities presented in Table 3. Comparing the continuum fluxes measured in the CFHT and WHT spectra in the 6760-7500 $\AA$ region, where the fringing in the CFHT spectrum is not too severe, the continuum flux in the WHT spectrum should be scaled upwards by a factor of 2.07 to match that in the CFHT spectrum. This scale factor is in good agreement with the value of 1.95 expected based upon the $1^{\prime \prime}$ slit used for the WHT spectra and the 1".22 seeing measured from the spatial profile of the continuum in the summed spectrum.

Generally, there is excellent agreement among the line intensities over the wavelength range $\mathrm{H} \delta$-He II $\lambda 5412$. In the nearultraviolet, the SPM spectrograph has very low efficiency and the upper limits we derive from those spectra are considerably less restrictive than the detections or limits from the CFHT spectrum. We give upper limits to the [S II] line intensities only for the last two SPM runs, since the CCD used for the first two SPM observing runs and that at CFHT suffered from fringing in the red.

The notable exception to the good agreement among the line intensities is $\mathrm{H} \alpha$. There is significant variation in the $\mathrm{H} \alpha / \mathrm{H} \beta$ ratio among the summed spectra for the different observing runs and between individual spectra for at least the
SPM2 and SPM4 observing runs. In both the SPM2 and SPM4 data sets, the dispersion among the $\mathrm{H} \alpha$ fluxes for the individual spectra also significantly exceeds that for the $\mathrm{H} \beta$ fluxes. In the last line of Table 2, we indicate the range of $\mathrm{H} \alpha / \mathrm{H} \beta$ values found among the individual spectra during each observing run. This variation is very puzzling, since we normally obtained all of the spectra consecutively on the same night (SPM2 is the exception). If this variation is real, it is occurring (irregularly) on a time scale of the order of an hour. Such behaviour is not at all expected in a nebular plasma (e.g., Aller 1987). In a typical SPM spectrum (of a half hour duration), at least 50 000, 15000 , and 2500 photons are detected at $\mathrm{H} \alpha, \mathrm{H} \beta$, and $\mathrm{H} \gamma$, respectively, so the variation we see in the $\mathrm{H} \alpha / \mathrm{H} \beta$ ratio would not appear to be due to poor photon statistics. Over this wavelength range, we do not see any variation exceeding more than a few percent in any of the standard star observations. We made no effort to orient the slit at the parallactic angle, but the wide slits used, particularly for the 2001 observing runs, should compensate for the effects of differential refraction. Regardless, were differential refraction the culprit, we should see nearly equally large variations in $\mathrm{H} \gamma / \mathrm{H} \beta$ as we see in $\mathrm{H} \alpha / \mathrm{H} \beta$ (Filippenko 1982), but we see none. Three instrumental effects, however, affect the 2001 data from SPM. First, the object was acquired by blind offset, so the centering of the object in the slit was almost certainly not optimal. Second, the offset guider is known 
Table 4. Log of the direct imaging observations.

\begin{tabular}{|c|c|c|c|c|c|}
\hline Telescope & Date & $\overline{C C C D}$ & Instrument & Filter $^{a}$ & Exposure time $^{b}$ \\
\hline SPM $2.1 \mathrm{~m}$ & 27-28 Мay 2001 & SITe $^{c}$ & Mexman & $\begin{array}{l}\mathrm{H} \alpha \quad(6565 \AA, 11 \AA) \\
\text { red cont. }(6650 \AA, 46 \AA)\end{array}$ & $\begin{array}{l}3900 \mathrm{~s}(5) \\
1500 \mathrm{~s}(3)\end{array}$ \\
\hline NOT $2.6 \mathrm{~m}$ & 1-2 June 2001 & $\mathrm{CCD} 7^{d}$ & ALFOSC & $\begin{array}{l}y \# 18 \quad(5470 \AA, 220 \AA) \\
H \alpha \# 21 \quad(6564 \AA, 33 \AA)\end{array}$ & $\begin{array}{l}2700 \mathrm{~s}(4) \\
2700 \mathrm{~s}(4)\end{array}$ \\
\hline
\end{tabular}

${ }^{a}$ The central wavelength and the bandpass width for each filter are given in parentheses.

${ }^{b}$ The number of images is given in parentheses.

${ }^{c}$ This CCD has $24 \mu \mathrm{m}$ pixels in a $1024 \times 1024$ format. Its gain and readnoise are $1.3 \mathrm{e}^{-} /$pix and $8 \mathrm{e}^{-}$, respectively. The plate scale is 0 '. $312 /$ pix.

${ }^{d}$ This CCD has $15 \mu$ m pixels in a $2048 \times 2048$ format. Its gain and readnoise are $\sim 1 \mathrm{e}^{-} /$pix and $6 \mathrm{e}^{-}$, respectively. The plate scale is $0{ }^{\prime} 188 /$ pix.

to flex relative to the instrument field of view, so the object centering was likely somewhat variable for the 2001 observations at SPM. Finally, the spectrograph was out of focus due the CCD being mis-aligned with the camera's focal plane. It is not clear, however, how any of these might introduce variations in the $\mathrm{H} \alpha / \mathrm{H} \beta$ ratio alone without affecting other line ratios. None of these issues affect the CFHT data nor those from SPM in 2002, yet the $\mathrm{H} \alpha / \mathrm{H} \beta$ variations exist in these data sets as well. Tovmassian et al. (2001) found similar variations, from a variety of observing sites, though they attributed them to the poor observing conditions affecting their observations. Although unusual, it would appear that the variations in $\mathrm{H} \alpha / \mathrm{H} \beta$ are real.

In Fig. 1, we compare the spectra of PNG 135.9+55.9 and the standard star G191B2B (Oke 1990). For PNG 135.9+55.9, we plot the CFHT spectrum for $\lambda<7500 \AA$ and the WHT spectrum for $\lambda>6760 \AA$, without applying any reddening correction to either spectrum. The WHT spectrum was scaled upwards by a factor of 2.07, as described previously. As noted by Tovmassian et al. (2001), this planetary nebula has a remarkably blue continuum.

Finally, these new data do not provide any direct diagnostic of the physical conditions in the nebular plasma. No density diagnostic has been detected to date, though [Ar Iv] $\lambda \lambda 4711,4740$ might have been expected given the high degree of ionization. Similarly, the only temperature diagnostic available is the upper limit to the [O $\mathrm{III}] \lambda 4363 / 5007$ ratio and it provides no useful constraint unless the density is unusually high, $10^{6}-10^{7} \mathrm{~cm}^{-3}$, which is excluded given the nebular flux and size (see Sect. 4).

\subsection{Reddening}

From the Schlegel et al. (1998) reddening maps, the expected foreground reddening is $E(B-V) \sim 01-0.02 \mathrm{mag}$. The $\mathrm{H} \gamma / \mathrm{H} \beta$ and $\mathrm{H} \delta / \mathrm{H} \beta$ ratios imply $E(B-V) \sim 0.3-0.35 \mathrm{mag}$, based upon a temperature of $2 \times 10^{4} \mathrm{~K}$, a density of $10^{3}-10^{4} \mathrm{~cm}^{-3}$, the Storey \& Hummer (1995) line emissivities, and the Fitzpatrick (1999) monochromatic reddening law parametrized with a ratio of total-to-selective extinction of 3.041 (McCall \& Armour 2000). On the other hand, the $\mathrm{H} \alpha / \mathrm{H} \beta$ ratio implies a reddening $E(B-V)<0.23$ mag for the same physical conditions, reddening law, and line emissivities, even if we consider the largest line ratio we observe, $\mathrm{H} \alpha / \mathrm{H} \beta=3.5$. For $\mathrm{H} \alpha / \mathrm{H} \beta$ ratios at the low end of the range observed, the reddening is zero or negative. The intensity of $\mathrm{P} 9 / \mathrm{H} \beta$ from the WHT spectrum implies $E(B-V)=0.05$ mag. We can also compute a reddening using He II $\lambda \lambda 4686,5412$. Adopting the same physical conditions, reddening law, and line emissivities, we find negative reddenings, i.e., He II $\lambda 4686$ is too bright relative to He II $\lambda 5412$ by $12 \%$. In any case, it appears that the reddening is at most modest, with $E(B-V)<0.3-0.35 \mathrm{mag}$. In the remainder of this paper, we shall assume that the reddening is zero. None of the conclusions would be affected had we adopted a modest reddening.

\section{Imaging}

\subsection{Observations}

We obtained direct images of PNG 135.9+55.9 in narrow-band filters from two sites (Table 4). Images were obtained in the emission line of $\mathrm{H} \alpha$ and a nearby continuum bandpass with the $2.1 \mathrm{~m}$ telescope in SPM. The Mexman filter wheel was used for observations. The seeing was modest, with stellar images having $F W H M$ of 1 '.5 or worse. Images were also obtained using ALFOSC at the $2.6 \mathrm{~m}$ Nordic Optical telescope (NOT) on La Palma, Canary Islands, Spain. A narrow filter centred at $\mathrm{H} \alpha$ was used, but the continuum was measured using a Stromgren $y$ filter. The NOT images benefited from better seeing, $\approx 1$ '. 2 , on the first night of this run. The spectrophotometric standard stars HZ44 and Feige 67 were observed along with the object on the second night for calibration purposes. During both imaging runs, sequences of at least 3 images in each filter were obtained, with one long exposure usually not exceeding $20 \mathrm{~min}$ and two shorter ones. This tactic allowed us to keep cosmic ray rate within reasonable limits in order to later combine the images for improved statistics and to permit the removal of cosmic rays.

The images were processed using IRAF. The steps included bias substraction, flat field correction, image combination, and apperture photometry. The STSDAS package within IRAF was used for the deconvolution of the images.

The primary uses of the $\mathrm{H} \alpha$ images were to determine the angular size of the nebula and to derive the density distribution of the gas. Two selected NOT images from the first night, where the seeing was better and the focus was good were combined to produce the image presented in Fig. 2. The nebula appears to be slightly elliptical in shape. No substructure is evident in the main body of the nebula. Its size comfortably exceeds the PSF of stellar objects, so the instrumental profile 

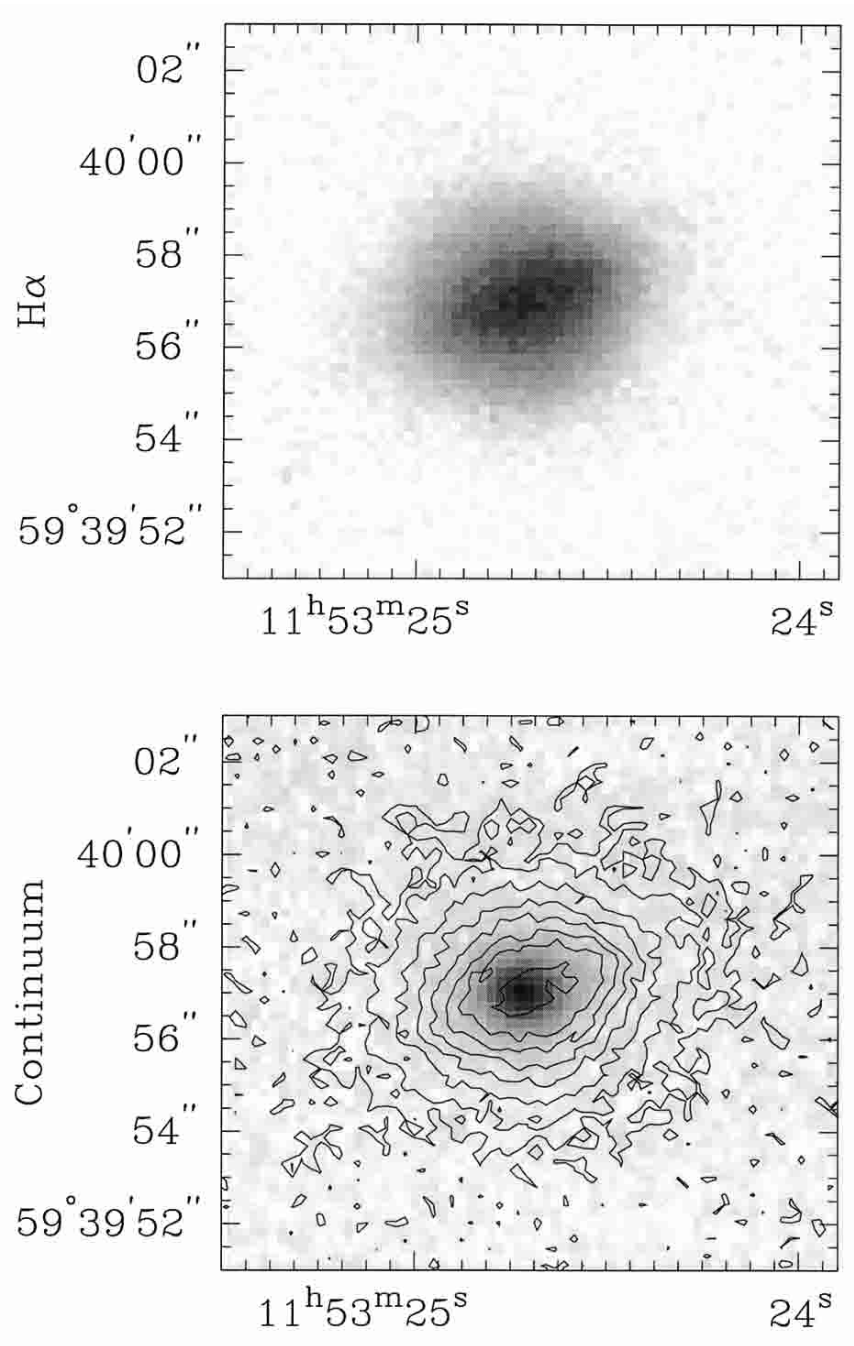

Fig. 2. The upper panel presents the image of PNG 135.9+55.9 in the light of $\mathrm{H} \alpha$, while the lower panel presents the Stromgren $y$ image of the central star with the $\mathrm{H} \alpha$ contours of the nebula superposed. The continuum and $\mathrm{H} \alpha$ images were aligned using field stars. Both images were obtained with the NOT.

should not affect its shape. Nonetheless, we conducted series of deconvolution experiments on the nebular images using the Lucy-Richardson method (Lucy 1974; Richardson 1972) that showed that the size and shape of the nebula were unchanged after deconvolution. This is our strongest evidence that the central star does not emit significantly in $\mathrm{H} \alpha$. For comparison the image of the central star obtained in the Stromgren $y$ filter is presented in the bottom panel of Fig. 2 with the $\mathrm{H} \alpha$ contours of the nebula superposed. We used the SPM observations, where the continuum filter was much closer in wavelength to the line filter, to substract the image of central stellar object from that of the nebula. Figure 3 presents the spatial profiles of the nebular emission in $\mathrm{H} \alpha$ from the NOT image without subtracting the central star and from the SPM image after substraction of central star. Except for a slight depression around the peak, the shape of the nebula in both observations is very similar.

The NOT observations in the Stromgren $y$ filter were used to estimate the magnitude of the central star. We inferred $m_{5556}=17.9 \pm 0.15 \mathrm{mag}$, corresponding to a flux of $F_{5556}=2.52 \times 10^{-16} \mathrm{erg} \mathrm{cm}^{-2} \mathrm{~s}^{-1} \AA^{-1}$ in a good agreement with the flux determined from spectroscopy. This continuum measurement should be reliable since there are almost no lines in the spectrum of this planetary nebula, the contribution of He II $\lambda 5413 \AA$ being negligible.

\subsection{The nebular angular diameter and density distribution}

The angular diameter of the planetary nebula is an important parameter since, in combination with the $\mathrm{H} \beta$ flux, it allows a determination of the electron density and the nebular mass. We followed the recipe of Bedding \& Zijlstra (1994) to assure that our measurements correspond to common standards. (For cautionary remarks, see van Hoof 2000.) There are a variety of definitions of the diameter of a PN in the literature: the FWHM, the surface brigthness contour at $10 \%$ of the maximum surface brightness, and the outermost contour at which emission is found. Since PNG 135.9+55.9 appears to have a slightly elliptical shape in the $\mathrm{H} \alpha$ images, we have measured the diameter of the nebula along two axes. We find that the diameter of the nebula is $11^{\prime \prime} 4 \times 9$.' 8 if we use the outermost contour, $6 . .3 \times 55^{\prime \prime} 2$ if we use the $10 \%$ surface brightness contour, and 3 ". $5 \times 2$ ". 95 if we use the $F W H M$.

Figure 4 shows the density and flux distributions adopted in our models. The symbols correspond to the observed $\mathrm{H} \alpha$ emission profiles across the major and minor axes obtained from the SPM images (shown in Fig. 3). The curve corresponds to a spherical model with a density distribution described by $n=n_{\mathrm{c}} \exp -(\theta / 2.8)^{2}$, where $\theta$ is the angular distance to the center in seconds of arc. In this model, the emissivity of $\mathrm{H} \alpha$ is taken to be simply proportional to $n^{2}$ and is computed for a uniform temperature. Such a simple representation accounts very satisfactorily for the observed $\mathrm{H} \alpha$ emission profile.

In the analysis of the photoionization models that follows, we adopt a nebular radius of 5.'6, i.e., similar to the diameter of the outermost contour.

\section{Photoionization analysis: New limits on the oxygen abundance}

\subsection{The modelling procedure}

The observational data do not permit a direct estimate of the oxygen abundance, since nothing is known of the electron temperature or of the presence of oxygen ions more charged than $\mathrm{O}^{++}$. It is therefore necessary to rely upon photoionization models.

We have constructed sequences of photoionization models in which the oxygen abundance varies over several orders of magnitude. The models are constrained by the available observations, which consist of the observed line intensities, the equivalent width of $\mathrm{H} \beta$, the total flux in $\mathrm{H} \beta$, the size of the nebula, and the radial distribution of $\mathrm{H} \alpha$ shown in Fig. 3. As already noted by Tovmassian et al. (2001), the shape of the stellar continuum only implies that the star is hotter than 


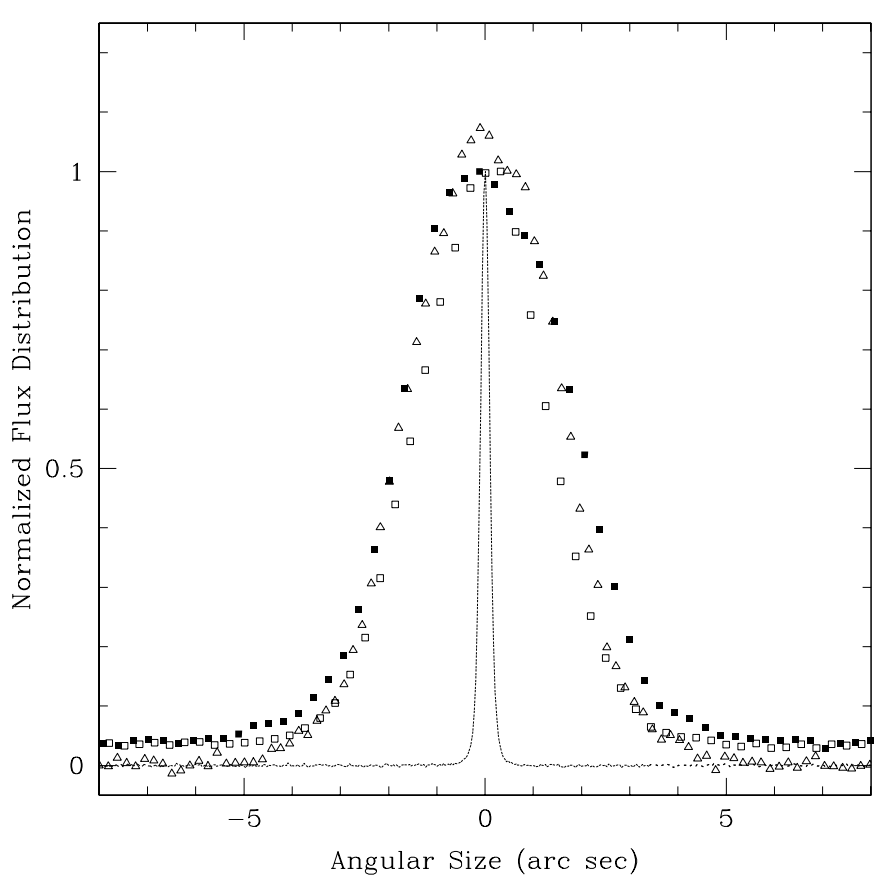

Fig. 3. This figure presents the spatial profiles of the nebula from the $\mathrm{H} \alpha$ images and of the central object from the $6650 \AA$ continuum image. The filled and open squares represent cuts along major and minor axes of the nebula from the SPM images after subtraction of the continuum. Open triangles represent the spatial profile along the major axis of the $\mathrm{H} \alpha$ image obtained at the NOT without continuum subtraction. The dotted line is the profile of the central star (SPM). The fluxes are normalized to the maximum intensity of the SPM continuumsubtracted image, except for the central star, which is normalized to its maximum intensity.

$50000 \mathrm{~K}$. The models are computed with the photoionization code PHOTO, using the atomic data listed in Stasińska \& Leitherer (1996). The central star is assumed to radiate as a blackbody of temperature $T_{\star}$. The hydrogen density at a radius $r$ is taken to be $n=n_{\mathrm{c}} \exp -(r / h)^{2}$, where $n_{\mathrm{c}}$ is a free parameter and $h=2.8 d / 2.06 \times 10^{5}$, where $d$, the distance to the nebula (in the same units as $h$ and $r$ ), is also a free parameter. The ionizing radiation field is treated in the outwardonly approximation. The computations start close to the star and are stopped when the equivalent width in $\mathrm{H} \beta, W(\mathrm{H} \beta)$, becomes equal to the observed value, taken to be $70 \AA$.

With such a representation of the nebula, it is easy to show that, for each assumed stellar temperature $T_{\star}$ and total luminosity $L_{\star}$, the total observed flux in $\mathrm{H} \beta$ implies a certain distance to the planetary nebula. The observed angular size of the image then fixes the outer radius of the nebula. By trial and error, we determine the value of $n_{\mathrm{c}}$ for which the radius $R_{\text {out }}$ corresponding to $W(\mathrm{H} \beta)=70 \AA$ is close to $5.6 d / 2.06 \times 10^{5}$. Table 5 gives some global properties of selected sequences of photoionization models. This includes the distance, $d$, and the height above the Galactic plane, $z$, implied by these models. Note that $R_{\text {out }}$ and the nebular mass, $M_{\text {neb }}$, are decreasing functions of the electron temperature. The values given in the table correspond to the low metallicity end of the model sequences.

The chemical abundances in each sequence of models are parametrized by $\mathrm{O} / \mathrm{H}$, with the abundances of the heavy
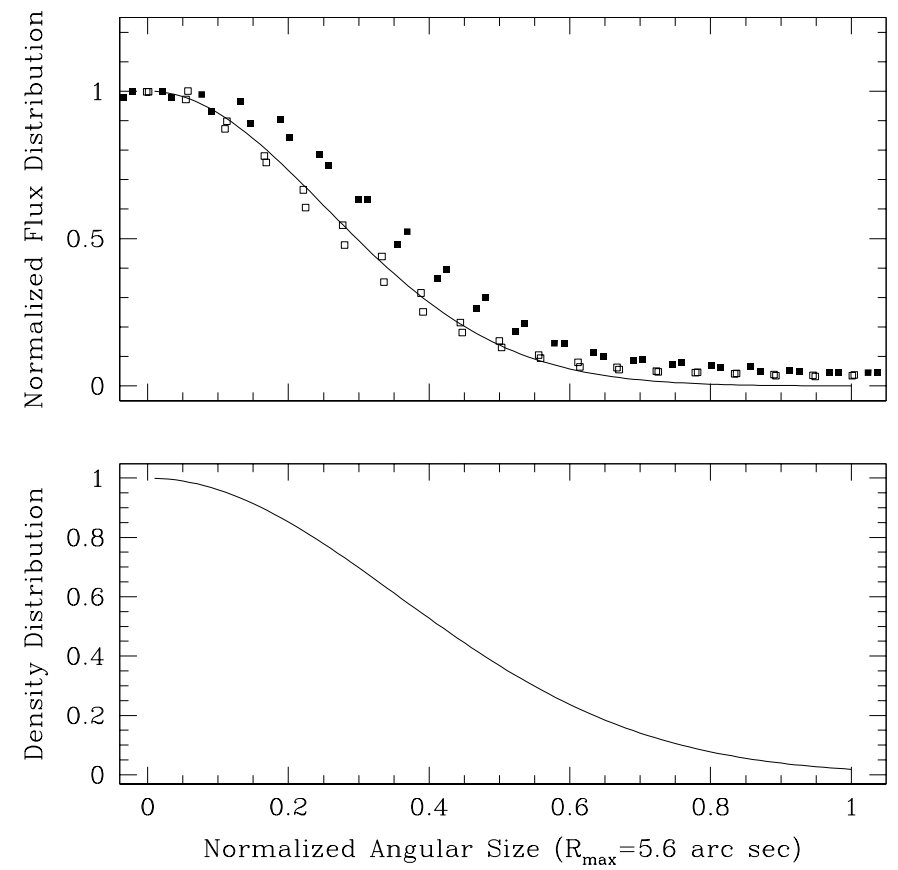

Fig. 4. The density distribution used for the model calculations is presented in the lower panel. The predicted flux distribution from our models is drawn with a solid line in the upper panel, supposing that the emissivity is proportional to $n^{2}$ and computed at a constant temperature. The open and filled squares denote the spatial distributions of the nebular emission along the major and minor axes, respectively, obtained from the SPM observations, as shown in Fig. 3. The abscissa denotes relative angular size normalized to the maximum, azimuthally-averaged radius of 5.' 6 .

elements with respect to oxygen following the recipe of McGaugh (1991). Note that the abundance ratios in PNG 135.9+55.9, as in any PN, may be significantly different from those assumed in McGaugh's recipe, especially for carbon and nitrogen. For helium, we assume an abundance of 0.08 with respect to hydrogen in all models. This is close to the value estimated from the observed HeII $4686 / \mathrm{H} \beta$ ratio and using the upper limit for He I $\lambda 5876$ given by the CFHT spectrum (see Sect. 5). In any event, our estimate of the chemical composition of PNG 135.9+55.9 is independent of the relative abundances adopted in the model sequences since our estimate uses the observed line intensities and relies on the ionization and temperature structure of the nebula, which depend essentially on hydrogen and helium in the domain of interest.

Figure 5 presents the results of our models as a function of $12+\log (\mathrm{O} / \mathrm{H})$. Each row of panels corresponds to a different central star temperature: $T_{\star}=100000 \mathrm{~K}, T_{\star}=125000 \mathrm{~K}$, and $T_{\star}=150000 \mathrm{~K}$, as indicated in the first panel. Each column of panels displays a different line ratio. In each panel, two series of models are represented with different symbols. Circles correspond to models with central stars having a total number of ionizing photons, $Q\left(\mathrm{H}^{0}\right)$, equal to $10^{47}$ photons s${ }^{-1}$, while squares correspond to models with a total number of ionizing photons of $6 \times 10^{47}$ photons $\mathrm{s}^{-1}$, values that roughly bracket the luminosities of the central stars of planetary nebulae as computed by post-AGB evolutionary models (Blöcker 1995). 
Table 5. Some global properties of the sequences of models investigated.

\begin{tabular}{cccccccc}
\hline \hline $\begin{array}{c}T_{\star} \\
{[\mathrm{K}]}\end{array}$ & $\begin{array}{c}Q\left(\mathrm{H}^{0}\right) \\
{\left[\mathrm{erg} \mathrm{s}^{-1}\right]}\end{array}$ & $\begin{array}{c}L(\mathrm{H} \beta) \\
{\left[\mathrm{erg} \mathrm{cm}^{-2} \mathrm{~s}^{-1}\right]}\end{array}$ & $\begin{array}{c}d \\
{[\mathrm{kpc}]}\end{array}$ & $\begin{array}{c}z \\
{[\mathrm{kpc}]}\end{array}$ & $\begin{array}{c}R_{\text {out }} \\
{[\mathrm{cm}]}\end{array}$ & $\begin{array}{c}M_{\text {neb }} \\
{\left[M_{\odot}\right]}\end{array}$ & $\begin{array}{c}n_{\mathrm{c}} \\
{\left[\mathrm{cm}^{-3}\right]}\end{array}$ \\
\hline 100000 & $6 \times 10^{47}$ & 0.48 & 25.0 & 20.7 & $1.8 \times 10^{18}$ & 0.78 & 125 \\
100000 & $1 \times 10^{47}$ & 0.080 & 10.2 & 8.5 & $6.3 \times 10^{17}$ & 0.070 & 190 \\
125000 & $6 \times 10^{47}$ & 0.29 & 19.2 & 15.9 & $1.6 \times 10^{18}$ & 0.45 & 148 \\
125000 & $1 \times 10^{47}$ & 0.048 & 7.8 & 6.5 & $6.1 \times 10^{17}$ & 0.044 & 223 \\
150000 & $6 \times 10^{47}$ & 0.20 & 16.0 & 13.3 & $1.3 \times 10^{18}$ & 0.29 & 163 \\
150000 & $1 \times 10^{47}$ & 0.032 & 6.5 & 5.4 & $4.8 \times 10^{17}$ & 0.027 & 245 \\
\hline
\end{tabular}

The horizontal lines show the observed values: thick lines for measured values or limits, thin lines indicating the uncertainties in measured values, and upward- and downward-pointing arrows denoting lower and upper limits, respectively. For the observational data, we adopted the CFHT observations, since they provide the most accurate measurements and the most stringent limits. For the $\mathrm{H} \alpha / \mathrm{H} \beta$ ratio, however, we plot the value derived from the SPM1 observations. As noted earlier, our observations give inconsistent results for the $\mathrm{H} \alpha / \mathrm{H} \beta$ ratio. Values of $\mathrm{H} \alpha / \mathrm{H} \beta$ around 3.1 are easily accounted for by our models, but lower values are extremely difficult to explain. We will return to this issue in Sect. 4.3.

\subsection{Discussion of the models}

The new observations allow us to eliminate the models with $T_{\star} \geq 125000 \mathrm{~K}$, since these produce a $[\mathrm{Ne} \mathrm{v}] /[\mathrm{Ne} \mathrm{III}]$ ratio much larger than is observed. Our models show that $T_{\star}$ should be around $100000 \mathrm{~K} . T_{\star}$ cannot be much lower than this value since He I $\lambda 5876$ would then be observed. Note that the observed lower limit to [O III]/[O II] provides no useful constraint upon the ionization structure (or central star temperature) of our models.

For the models with $T_{\star}=100000 \mathrm{~K}$ shown in Fig. 5, the oxygen abundances compatible with the observed $[\mathrm{O}$ III] $/ \mathrm{H} \beta$ ratio are $5.8<12+\log \mathrm{O} / \mathrm{H}<6.3 \mathrm{dex}$. Allowing for a reasonable uncertainty in $T_{\star}$ implies $12+\log \mathrm{O} / \mathrm{H} \leq 6.5 \mathrm{dex}$. The upper limit to $[\mathrm{Ne} \mathrm{v}] /[\mathrm{Ne}$ III] eliminates models excited by a star with a temperature significantly above $100000 \mathrm{~K}$ which would otherwise permit $12+\log \mathrm{O} / \mathrm{H}$ of the order of 7-8 dex.

We have constructed other series of models to test the robustness of these conclusions. For example, we have calculated models in which the nebular radius, total nebular flux, and $\mathrm{H} \beta$ equivalent width were varied, but the conclusions remain unchanged.

We have also considered models in which the gas is distributed in small clumps with the same global density law as above, but with an overall filling factor of 0.1. Although the $\mathrm{H} \alpha$ image is smooth at our resolution, we cannot a priori exclude the presence of small scale clumps or filaments. Clumpy models that fit the observational constraints will result in a lower global ionization level than smoothlydistributed models. For the purposes of illustration, Fig. 6 compares the smooth model of Fig. 5 with $T_{\star}=150000 \mathrm{~K}$ and
$Q\left(\mathrm{H}^{0}\right)=10^{47} \mathrm{ph} \mathrm{s}^{-1}$ (circles) with a clumpy model whose filling factor is 0.1 (squares). Because the ionization is lower in the clumpy model, the $\mathrm{O}^{+++} / \mathrm{O}^{++}$ratio is lowered with respect to the smooth model, while the $[\mathrm{O}$ III $] \lambda 5007 / \mathrm{H} \beta$ ratio is raised. Consequently, the oxygen abundance compatible with the observed [O III] $\lambda 5007 / \mathrm{H} \beta$ ratio is smaller than in the smooth case. Note that the model presented here gives $12+\log \mathrm{O} / \mathrm{H} \simeq$ $6.5 \mathrm{dex}$, but still violates the [Ne v] $\lambda 3426 /[\mathrm{Ne}$ III] $\lambda 3869$ and $\mathrm{H} \alpha / \mathrm{H} \beta$ constraints. The total nebular mass of a model of given total $\mathrm{H} \beta$ luminosity and given radius is roughly proportional to the square of the filling factor. Therefore, clumpy models that satisfy the observational constraints will have lower nebular masses than the corresponding models listed in Table 5. Finally, if the density in the clumps were extremely high, models with high metallicities, even as high as solar, could account for the weak intensities of the forbidden lines, because these lines would be quenched by collisional de-excitation. For this to occur, densities exceeding $10^{6} \mathrm{~cm}^{-3}$ are required for both [O III] $\lambda 5007$ and [ $\mathrm{Ne}$ III] $\lambda 3869$. Given that the $\mathrm{H} \beta$ flux and equivalent width as well as the size of the nebula are known, such high densities would imply that the filling factor would have to be of the order of $10^{-8}$ or less, which is highly unrealistic.

Our computations were made assuming that the star radiates as a blackbody. However, a more realistic stellar atmosphere would give a different spectral energy distribution for the ionizing photons, particularly at the largest energies. One expects that extended, metal-poor atmospheres could have larger fluxes at energies above $100 \mathrm{eV}$. This would increase the $[\mathrm{Ne} \mathrm{v}] /[\mathrm{Ne} \mathrm{III}]$ ratio and consequently strenghten our conclusion that $12+\log \mathrm{O} / \mathrm{H} \leq 6.5 \mathrm{dex}$. On the other hand, absorption by metals could depress the number of photons able to produce [Ne v] (Rauch 2002), in which case a star with $T_{\star} \sim 150000 \mathrm{~K}$ could become acceptable. In this case, however, the excitation of the nebula would be lower than that predicted by blackbody models with $T_{\star}=150000 \mathrm{~K}$ and the line ratios would resemble those produced by the blackbody model with $T_{\star}=100000 \mathrm{~K}$, again implying $12+\log \mathrm{O} / \mathrm{H} \leq 6.5 \mathrm{dex}$. In any case, the amount of metals in the atmosphere is not expected to be large, unless the atmosphere contains dredged-up carbon. A definitive answer to this problem can only come from a direct measurement of lines from more highly charged ions.

Note that the distance we obtain for our object (see Table 5) indicates that it is located in the Galactic halo, in agreement 

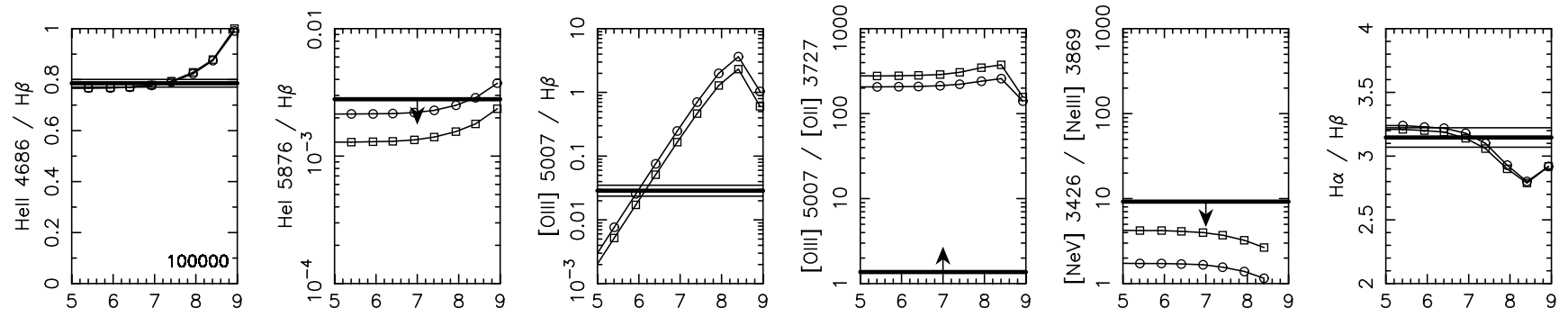

$12+\log (\mathrm{O} / \mathrm{H})$
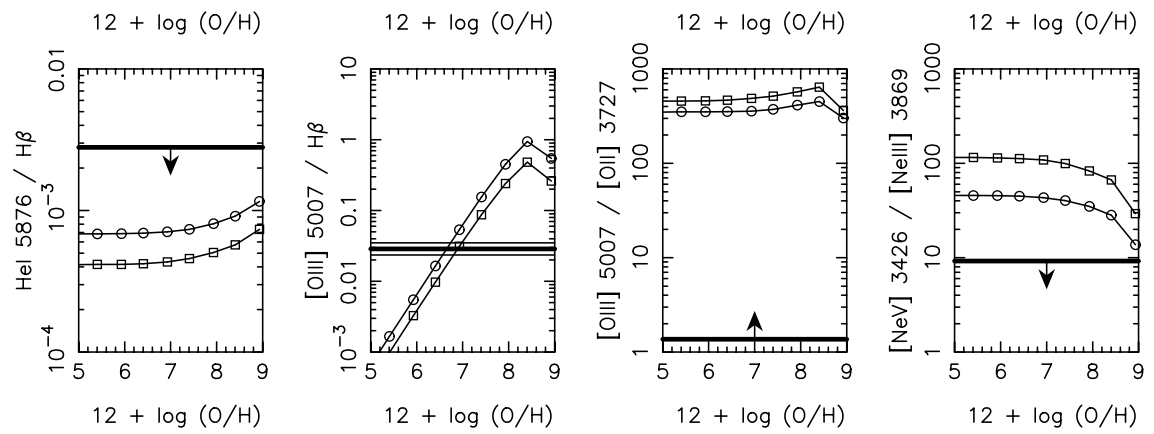

$12+\log (\mathrm{O} / \mathrm{H})$
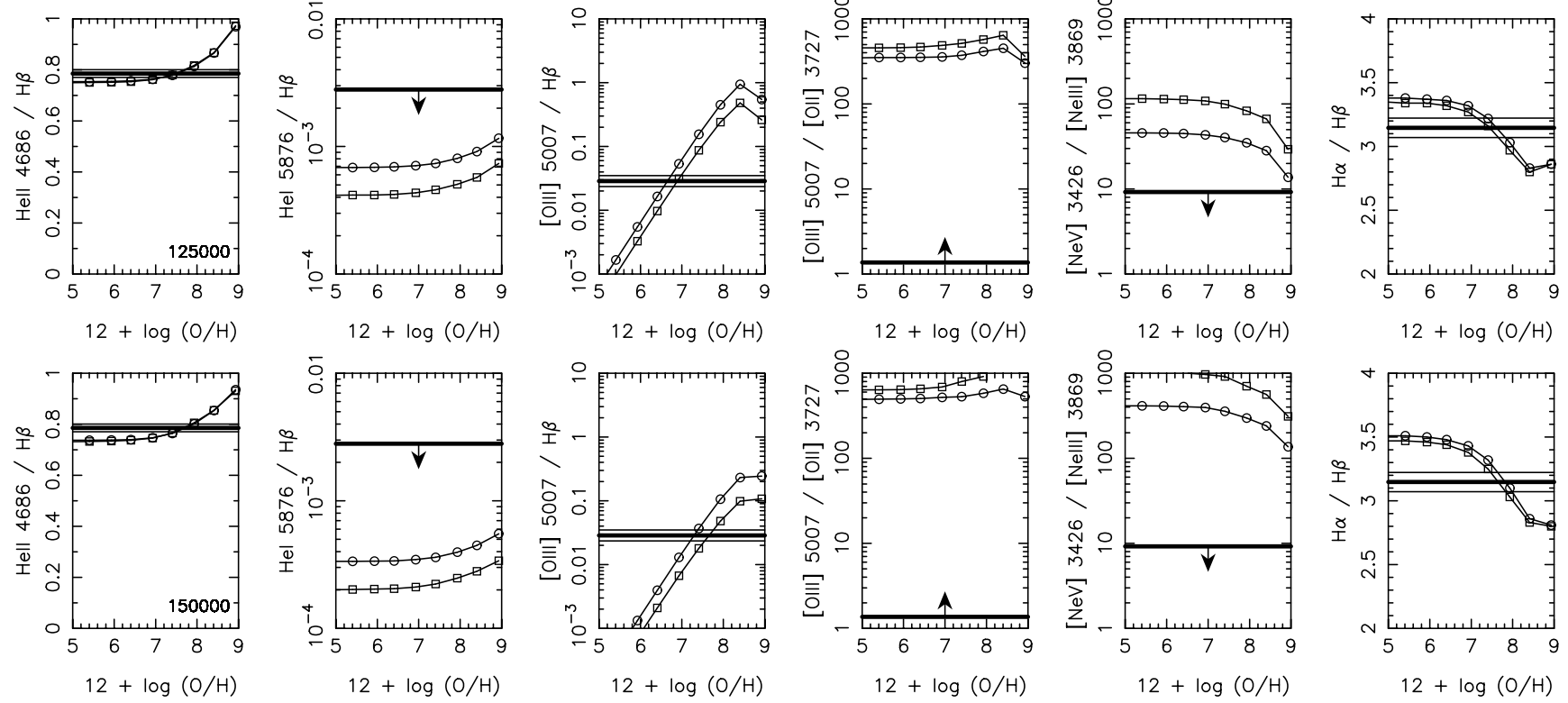

Fig. 5. This figure presents our grid of photoionization models. Each row of panels corresponds to a particular central star temperature, indicated in the first panel. Each column of panels plots a different line intensity ratio. In each panel, two sequences of models are included: circles denote models with ionizing luminosities of $10^{47}$ photons s $\mathrm{s}^{-1}$, squares denote models with ionizing luminosities of $6 \times 10^{47}$ photons s${ }^{-1}$. The thick horizontal lines indicate the observed values or their limits from the CFHT spectrum. In the case of limits, upward- and downward-pointing arrows denote whether they are lower or upper limits, respectively. Thin horizontal lines denote the uncertainty range of measured values.
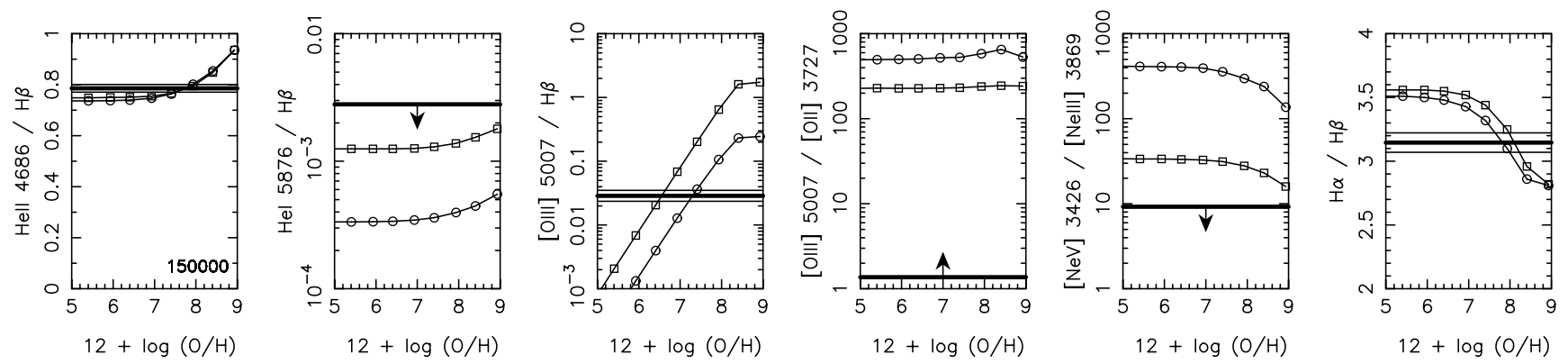

Fig. 6. Here, we compare two models with $T_{\star}=150000 \mathrm{~K}$ and $Q\left(\mathrm{H}^{0}\right)=10^{47}$ photon $\mathrm{s}^{-1}$, but with the circles denoting a filling factor of 1 (the model from Fig. 5) and squares denoting a filling factor of 0.1. As this experiment demonstrates, the maximum oxygen abundance allowed by the models decreases as the filling factor decreases.

with its radial velocity (Tovmassian et al. 2001). Its derived nebular mass is compatible with the range of nebular masses derived for Magellanic Cloud PNe (Barlow 1987).

To conclude this section, we emphasize that our new observational data allow us to confirm that PNG $135.9+55.9$ is an extremely oxygen-poor planetary nebula, with an oxygen abundance less than $1 / 50$ of the solar value. Our models favour a value of $12+\log \mathrm{O} / \mathrm{H}$ between 5.8 and $6.5 \mathrm{dex}$, compared with the solar value of $12+\log \mathrm{O} / \mathrm{H}=8.83$ dex (Grevesse \& Sauval 1998). Our modelling experiments indicate that this conclusion is independent of plausible changes in the properties assumed for the central star or nebular envelope.

\subsection{The $H \alpha / H \beta$ problem}

Our preferred models with $T_{\star}=100000 \mathrm{~K}$ and low metallicities are compatible with the $\mathrm{H} \alpha / \mathrm{H} \beta$ ratio derived from the 
SPM1 data, as well as with the highest values found in individual spectra from the other SPM observations (Table 2, Fig. 5). However, as discussed earlier, we find apparently significant variations of the $\mathrm{H} \alpha / \mathrm{H} \beta$ ratio between the different observing runs and even among individual spectra obtained during individual runs. The majority of the individual spectra indicate that $\mathrm{H} \alpha / \mathrm{H} \beta$ is below 3, as do the observations reported by Tovmassian et al. (2001).

Because of collisional excitation of the hydrogen lines, $\mathrm{H} \alpha / \mathrm{H} \beta$ cannot have the recombination value, but is expected to be larger. Collisional excitation is unavoidable at electron temperatures above $\sim 15000 \mathrm{~K}$ as soon as there is a small fraction of residual neutral hydrogen. The amount by which $\mathrm{H} \alpha / \mathrm{H} \beta$ exceeds the recombination value $(2.75$ at $20000 \mathrm{~K}, 2.70$ at $30000 \mathrm{~K}$, using the case B coefficients of Storey \& Hummer 1995) depends upon both the electron temperature, which is higher for higher values of $T_{\star}$, and on the amount of neutral hydrogen. For example, the models with $T_{\star}=125000 \mathrm{~K}$ predict a value of $\mathrm{H} \alpha / \mathrm{H} \beta$ around 3.4 at low metallicities. With our observational constraints, there is not much room for a drastic reduction of the proportion of neutral hydrogen in our models. Indeed, for a given $T_{\star}$ and chemical composition, the proportion of $\mathrm{H}^{0}$ at each point in the nebula is completely determined by (and roughly proportional to) $n_{\mathrm{e}} /\left(L_{V} / R^{2}\right)$, where $n_{\mathrm{e}}$ is the local electron density, $L_{V}$ is the stellar luminosity in the $V$ band and $R$ is the distance of this point to the star. $L_{V} / R^{2}$ is a distance-independent quantity that relates the stellar flux in the $V$ band to the angular distance of this point to the star; $n_{\mathrm{e}}$ is determined by the density distribution law obtained from our $\mathrm{H} \alpha$ images and the value of $n_{\mathrm{c}}$ is imposed by the observational constraints on $W(\mathrm{H} \beta)$ and the total angular radius, as explained in Sect. 4.1. Our models of PNG 135.9+55.9 with $T_{\star}=100000 \mathrm{~K}$ indicate that the $\mathrm{H} \alpha / \mathrm{H} \beta$ ratio should not be lower than 3 if the nebula is metal-poor. Values of $T_{\star}$ significantly below $100000 \mathrm{~K}$ could be consistent with some of the observed values of $\mathrm{H} \alpha / \mathrm{H} \beta$ but they are excluded by the failure to observe He I $\lambda 5876$ in this object.

Does this mean that the object is not as oxygen-poor as inferred above? There are several arguments against a higher abundance. In our $T_{\star}=100000 \mathrm{~K}$ models, $\mathrm{H} \alpha / \mathrm{H} \beta<3$ implies $12+\log \mathrm{O} / \mathrm{H}>7.5 \mathrm{dex}$, but this is clearly incompatible with the observed value of [O $\mathrm{III}] \lambda 5007 / \mathrm{H} \beta$, as seen in Fig. 5. At the other extreme, for our $T_{\star}=150000 \mathrm{~K}$ models, $\mathrm{H} \alpha / \mathrm{H} \beta<3$ implies $12+\log \mathrm{O} / \mathrm{H}>8 \mathrm{dex}$, which, though marginally compatible with the observed [O III] $\lambda 5007 / \mathrm{H} \beta$, predicts a value for the [ $\mathrm{Ne} \mathrm{v}] \lambda 3426 /[\mathrm{Ne}$ III] $\lambda 3869$ ratio far larger than observed.

Nor is it likely that any possible variability of the $\mathrm{H} \alpha / \mathrm{H} \beta$ ratio affects our oxygen abundances. Supposing that this variation is real and refers entirely to the nebular radiation, one would then expect the [O III] $\lambda 5007 / \mathrm{H} \beta$ ratio to vary as a consequence of variable ionization or temperature conditions, but this is not seen. All of the line intensities apart from $\mathrm{H} \alpha$ remain remarkably constant, including the [O III] $\lambda 5007$ line. Therefore, we believe that the $\mathrm{H} \alpha / \mathrm{H} \beta$ problem does not affect our conclusions as regards the oxygen abundance in PNG 135.9+55.9.

However, this $\mathrm{H} \alpha / \mathrm{H} \beta$ problem is extremely puzzling. One does not expect this ratio to vary in nebular conditions for an extended object. One possibility could be that PNG 135.9+55.9 harbours a compact disk. Such a suggestion has been made for other planetary nebulae based upon either morphological, spectroscopic, or variability arguments (He 2-25: Corradi 1995; IC 4997: Miranda \& Torrelles 2000; Lee \& Hyung 2000; and M 2-9: Livio \& Soker 2001). Accretion disks in close, interacting binary systems have the particularity of both being variable (Warner 1995) and having $\mathrm{H} \alpha / \mathrm{H} \beta$ ratios much smaller than 3 , sometimes attaining values below unity (e.g., Williams 1995). If PNG 135.9+55.9 contained such an accretion disk and if this disk contributed to the emission of the hydrogen and helium lines in the central part of the nebula, this could explain both the variability of the $\mathrm{H} \alpha / \mathrm{H} \beta$ ratio in $\mathrm{PNG} 135.9+55.9$ and the values of 2.7 or lower observed in some of our spectra. However, an accretion disk would be an unresolved point source in our images, and our deconvolution experiments found no significant contribution to the $\mathrm{H} \alpha$ emission from the central source. Likewise, the lack of Dopper-broadened emission lines (Tovmassian et al. 2001) also argues against an accretion disk as the origin of a significant fraction of the line emission (Warner 1995).

\section{The abundances of the remaining elements}

Our high signal-to-noise spectra enabled us to measure the intensity of the [Ne III] $\lambda 3869$ line, and to derive upper limits to the intensities of [S III] $\lambda 9069$ and [Ar III] $\lambda 7135$, which allow us to make some inferences on the abundances of $\mathrm{Ne}, \mathrm{S}$ and $\mathrm{Ar}$.

To compute abundances, we must rely upon the electron temperature and ionization structure provided by our models. The relevant quantities are shown in Fig. 7 for the models from Fig. 5. The first column of panels on the left repeats the [O III] $\lambda 5007 / \mathrm{H} \beta$ ratio from Fig. 7 for reference. The second column of panels gives the value of $T\left(\mathrm{O}^{++}\right)$defined as:

$T\left(\mathrm{O}^{++}\right)=\frac{\int T_{\mathrm{e}} n\left(\mathrm{O}^{++}\right) n_{\mathrm{e}} \mathrm{d} V}{\int n\left(\mathrm{O}^{++}\right) n_{\mathrm{e}} \mathrm{d} V}$.

The next columns of panels give the ionization fractions $x\left(\mathrm{O}^{++}\right) / x\left(\mathrm{H}^{+}\right), \quad x\left(\mathrm{O}^{++}\right) / x\left(\mathrm{Ne}^{++}\right), \quad x\left(\mathrm{O}^{++}\right) / x\left(\mathrm{~S}^{++}\right)$and $x\left(\mathrm{O}^{++}\right) / x\left(\mathrm{Ar}^{++}\right)$, where the notation $x\left(\mathrm{O}^{++}\right)$stands for:

$x\left(\mathrm{O}^{++}\right)=\frac{\int n\left(\mathrm{O}^{++}\right) n_{\mathrm{e}} \mathrm{d} V}{\int n(\mathrm{O}) n_{\mathrm{e}} \mathrm{d} V}$.

The value of $x\left(\mathrm{O}^{++}\right) / x\left(\mathrm{H}^{+}\right)$is shown here simply to demonstrate that $\mathrm{O}^{++}$is far from being the dominant oxygen ion in the nebula, and that a more robust determination of the oxygen abundance would require observing lines from higher ionization stages.

Using the same atomic data as in the photoionization code and taking as a characteristic temperature of the emission of the [Ne III] $\lambda 3869$ and [O III] $\lambda 5007$ lines a value of $30000 \mathrm{~K}$ derived from our preferred models (Fig. 5), we find that $\mathrm{Ne}^{++} / \mathrm{O}^{++}=0.70 \pm 0.4$, where the uncertainty reflects the uncertainties of the [O III] $\lambda 5007$ and [Ne III] $\lambda 3869$ line intensities. We furthermore note that both $\mathrm{O}^{++}$and $\mathrm{Ne}^{++}$are minority ions (Fig. 7). Generally, one adopts $\mathrm{Ne}^{++} / \mathrm{O}^{++}=\mathrm{Ne} / \mathrm{O}$. In our preferred models (those corresponding to $T_{\star}=100000 \mathrm{~K}$ ), we 

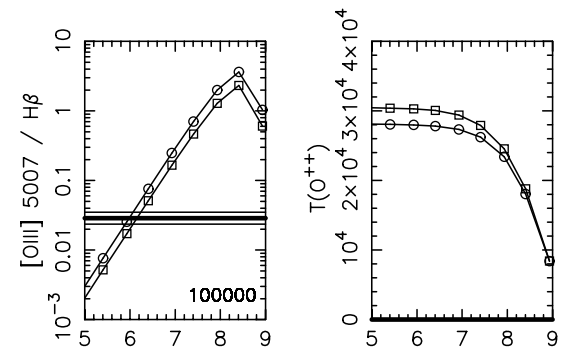

$12+\log (\mathrm{O} / \mathrm{H})$
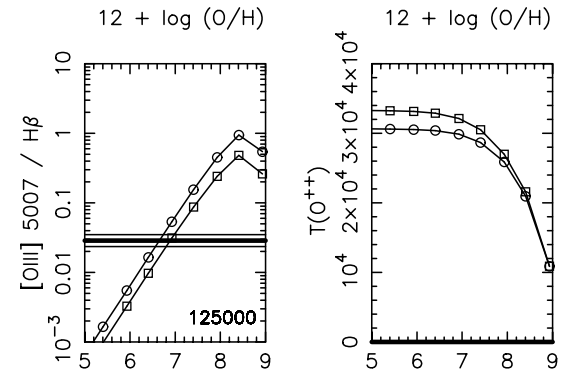

$12+\log (\mathrm{O} / \mathrm{H})$
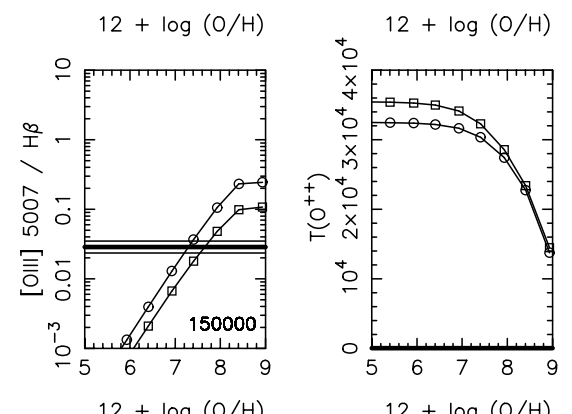

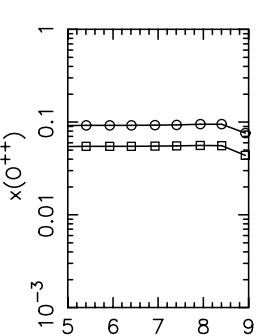

$12+\log (\mathrm{O} / \mathrm{H})$

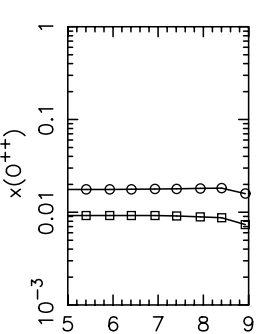

$12+\log (\mathrm{O} / \mathrm{H})$

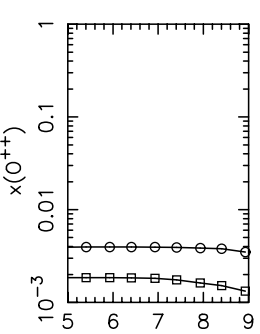

$12+\log (\mathrm{O} / \mathrm{H})$
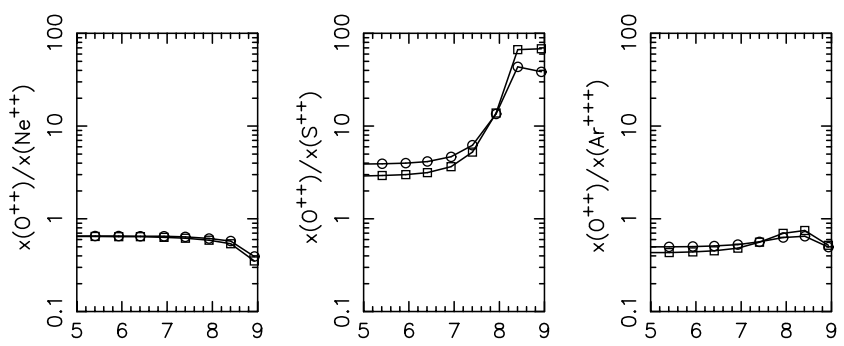

$12+\log (\mathrm{O} / \mathrm{H})$
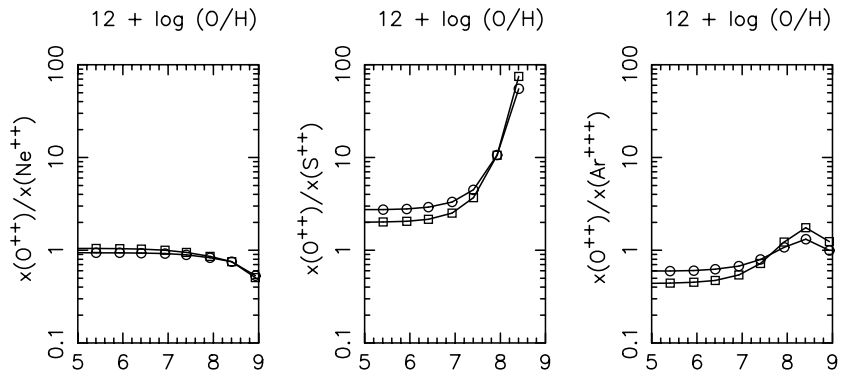

$12+\log (\mathrm{O} / \mathrm{H})$
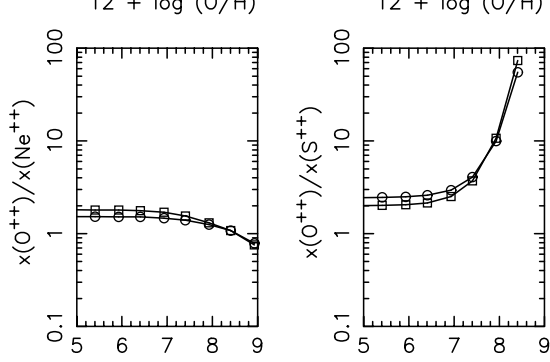

$12+\log (\mathrm{O} / \mathrm{H})$

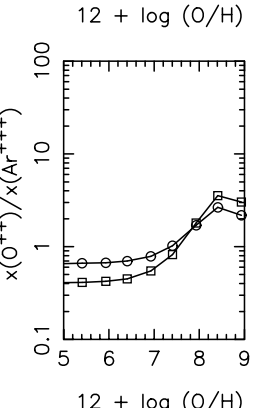

Fig. 7. This figure presents various physical parameters for the grid of models in Fig. 5. From left to right, the columns present [O III] $\lambda 5007 / \mathrm{H} \beta$, the temperature in the $\mathrm{O}^{++}$zone, the weighted fraction of oxygen in the form of $\mathrm{O}^{++}$, and the ionization correction factors for $\mathrm{Ne}^{++}, \mathrm{S}^{++}$, and $\mathrm{Ar}^{+++}$with respect to $\mathrm{O}^{++}$. These data are required to derive the abundances of $\mathrm{Ne}, \mathrm{S}$, and $\mathrm{Ar}$ in $\mathrm{PNG}$ 135.9+55.9.

find from Fig. 7 that the ionization correction factor is rather around 0.7 , thus leading to $\mathrm{Ne} / \mathrm{O}=0.5 \pm 0.3$.

In a similar fashion, our observed limits on the intensities of the $[\mathrm{Ar}$ Iv] $\lambda \lambda 4711,4740$ lines allow us to derive that $\mathrm{Ar}^{+++} / \mathrm{O}^{++}<0.45$. From our models (Fig. 7) we find that the ionization correction factor is 0.5 , and therefore $\mathrm{Ar} / \mathrm{O}<0.23$.

Given that the nebula is strongly density-bounded, the observed limits on the [N II] $\lambda 6584$ and [S II] $\lambda 6716, \lambda 6731$ line intensities give only crude limits on abundance ratios involving these elements. With the ionization correction factors from our preferred model, we find $\mathrm{N} / \mathrm{H}<2.3 \times 10^{-5}$ and $\mathrm{S} / \mathrm{H}<7 \times 10^{-5}$. Such limits are not very useful, except to infer that the N/H ratio is at most equal to that in the Orion nebula.

Our infrared spectra allow us to estimate upper limits to the intensities of [S III] $\lambda 9069$ and [Ar III] $\lambda 7135$, which are respectively $8 \times 10^{-4}$ and $6 \times 10^{-4}$ of the intensity of $\mathrm{H} \beta$. These upper limits imply that $\mathrm{S}^{++} / \mathrm{O}^{++}<0.031$ and $\mathrm{Ar}^{++} / \mathrm{O}^{++}<0.012$. In our preferred photoionization models, the ionization correction factor to derive $\mathrm{Ar} / \mathrm{O}$ from $\mathrm{Ar}^{++} / \mathrm{O}^{++}$is around 20, so that the upper limit on [Ar III] $\lambda 7135$ implies $\mathrm{Ar} / \mathrm{O}<0.24$ (in agreement with the upper limit derived from [Ar Iv] $\lambda \lambda 4711,4740$ ). Regarding sulfur, we find that the ionization correction factor is around 3 for $\mathrm{S} / \mathrm{O}$ from our models (Fig. 7), implying that $\mathrm{S} / \mathrm{O}<0.094$. It must be noted that the atomic data concern-
Table 6. $\alpha$-element abundance ratio comparison with other objects.

\begin{tabular}{|c|c|c|c|}
\hline object & $\overline{\mathrm{Ne} / \mathrm{O}}$ & $\mathrm{S} / \mathrm{O}$ & $\mathrm{Ar} / \mathrm{O}$ \\
\hline PNG $135.9+55.9^{a}$ & $0.5 \pm 0.3$ & $<0.094$ & $<0.23$ \\
\hline Galactic disk $\mathrm{PNe}^{b}$ & 0.26 & 0.017 & 0.005 \\
\hline Galactic halo $\mathrm{PNe}^{c}$ & 0.13 & 0.016 & 0.0016 \\
\hline Orion nebula ${ }^{d}$ & 0.18 & 0.03 & 0.014 \\
\hline $\operatorname{Sun}^{e}$ & 0.18 & 0.03 & 0.004 \\
\hline
\end{tabular}

${ }^{a}$ This work.

${ }^{b}$ Kingsburgh \& Barlow (1994).

${ }^{c}$ Howard et al. (1997). These authors also show that the abundance ratios are more dispersed in halo $\mathrm{PNe}$ than in disk $\mathrm{PNe}$.

${ }^{d}$ Esteban et al. (1998).

${ }^{e}$ Grevesse \& Sauval (1998).

ing the ionization structure of S and Ar are uncertain (see e.g. Ferland et al. 1998). Our models, were computed without including dielectronic recombination to low-lying levels for these ions. It is likely that the real ionization fractions of $\mathrm{S}^{++}$and $\mathrm{Ar}^{+++}$are actually higher than predicted by the models, giving smaller ionization correction factors and more stringent limits.

It is interesting to compare the ratios of $\mathrm{Ne} / \mathrm{O}, \mathrm{S} / \mathrm{O}$ and $\mathrm{Ar} / \mathrm{O}$ we find for PNG 135.9+55.9 with those of other kinds 
of objects. Table 6 shows the values for a sample of PNe in the Galactic disk (Kingsburgh \& Barlow 1994) and in the Galactic halo (Howard et al. 1997), for the Orion nebula (Esteban et al. 1998), and for the Sun (Grevesse \& Sauval 1998). This table is of course subject to uncertainties. Even the solar abundances are quite uncertain for $\mathrm{Ne}$, Ar and $\mathrm{O}$ (see Grevesse \& Sauval 1998; the recent oxygen abundance determination from Allende Prieto et al. 2001 yields a value that is only $73 \%$ that obtained by Grevesse \& Sauval 1998). One might then argue that the $\mathrm{Ne} / \mathrm{O}$ ratio in $\mathrm{PNG} 135.9+55.9$ is compatible with the solar value, but we note that it is about twice the value found in the Orion nebula and in disk planetary nebulae, where the systematic errors in the abundance derivations are likely to be similar. Supposing our $\mathrm{Ne} / \mathrm{O}$ ratio is corect, it might indicate some conversion of $\mathrm{O}$ into $\mathrm{Ne}$ by $\alpha$ capture. A few similar cases are known among planetary nebulae (e.g., BB-1 has a $\mathrm{Ne} / \mathrm{O}=$ 0.77; Howard et al. 1997). Another possibility is that the material from which the progenitor of PNG 135.9+55.9 formed had an anomalous Ne/O ratio. Abundance studies of metalpoor stars indicate that the very early galaxy was chemicallyinhomogeneous, with individual sites of star formation being influenced by the explosions of nearby supernovae (e.g., Burris et al. 2000). The yields of $\mathrm{O}$ and $\mathrm{Ne}$ from individual supernovae are also a function of the stellar mass (Woosley \& Weaver 1995; Thielemann et al. 1996), while observations of metalpoor halo stars indicate that the scatter in oxygen abundances is $0.3-0.5 \mathrm{dex}$ at very low oxygen abundances (e.g., Israelian et al. 2001). As a result, it is probably not surprising that the progenitor of PNG 135.9+55.9 might not have formed out of material with the same $\mathrm{Ne} / \mathrm{O}$ ratio that has characterized the more recent Galactic disk. Regardless, any conversion of $\mathrm{O}$ into $\mathrm{Ne}$ is at most modest. Even were all of the $\mathrm{Ne}$ formed by nuclear processing from oxygen, the initial oxygen abundance would have been only 0.18 dex larger than our preferred values. Thus, the extremely low oxygen abundance in PNG 135.9+55.9 is genuine and not due to nuclear and mixing processes in the progenitor star. The oxygen abundance in PNG 135.9+55.9 should consequently reflect the chemical composition of the medium out of which the star was made. The limits we obtain on $\mathrm{S} / \mathrm{O}$ and $\mathrm{Ar} / \mathrm{O}$ in our object are consistent with this view (although the limits are not very stringent).

The accuracy of the helium line intensities achieved in the present observations raised the hope of obtaining very accurate helium abundances. Taking the intensities determined from our CFHT observations and adopting a temperature of $30000 \mathrm{~K}$ as inferred from our models and case B coefficients from Storey $\&$ Hummer (1995) we find that $\mathrm{He}^{++} / \mathrm{H}^{+}=(7.50 \pm 0.14) \times 10^{-2}$. The upper limit to the intensity of $\mathrm{He}$ I $\lambda 5876$ in the same spectra gives an upper limit to $\mathrm{He}^{+} / \mathrm{H}^{+}$of $0.24 \times 10^{-2}$ when using the emissivities from Benjamin et al. (1999) in the low density limit. A proper determination of the uncertainty in the derived helium abundance should, however, account for collisional excitation of the lines, a possible small amount of reddening, possible deviations from case B, possible underlying absorption, as well as temperature gradients inside the nebula. All of this can only be attempted once the $\mathrm{H} \alpha / \mathrm{H} \beta$ problem is solved. It is therefore premature to propose an accurate value for the helium abundance in PNG 135.9+55.9.

\section{Conclusions}

Our new, extensive observations of PNG 135.9+55.9 confirm its unusual nature. Spectroscopy covering the wavelength interval 3400-9700 A reveals lines of only H I, He II, [O III], and [Ne III]. Deep $\mathrm{H} \alpha$ imaging was used to derive the radial density distribution.

Using these data as constraints, we constructed a new set of nebular models from which we derived the abundance of oxygen and the $\mathrm{Ne} / \mathrm{O}, \mathrm{S} / \mathrm{O}$, and $\mathrm{Ar} / \mathrm{O}$ abundance ratios. We confirm the extremely low value of the oxygen abundance, which we find to be less than $1 / 50$ of the solar value: our models favour a value of $12+\log \mathrm{O} / \mathrm{H}$ between 5.8 and $6.5 \mathrm{dex}$. The distance implied by these models places PNG 135.9+55.9 in the Milky Way halo, in accordance with its radial velocity (Tovmassian et al. 2001). The models also imply nebular masses in the range expected.

For the $\alpha$-element ratios, we find $\mathrm{Ne} / \mathrm{O}=0.5 \pm 0.3$, $\mathrm{S} / \mathrm{O}<0.094$, and $\mathrm{Ar} / \mathrm{O}<0.23$. The $\mathrm{Ne} / \mathrm{O}$ ratio may be somewhat higher than is commonly found in planetary nebulae in the Milky Way disk (e.g., Henry 1989; Kingsburgh $\&$ Barlow 1994). One possibility is that the progenitor of PNG 135.9+55.9 converted some of its $\mathrm{O}$ to $\mathrm{Ne}$. It is also possible that the anomalous $\mathrm{Ne} / \mathrm{O}$ ratio is the result of discrete chemical enrichment in the very early evolution of the galaxy (e.g., Burris et al. 2000). Regardless, of the cause, any conversion of $\mathrm{O}$ to $\mathrm{Ne}$ has been modest and does not affect our conclusion that PNG $135.9+55.9$ is the progeny of an intrinsically very oxygen-poor star.

An unusual characteristic of PNG $135.9+55.9$ is its low $\mathrm{H} \alpha / \mathrm{H} \beta$ ratio, for which we find no clear explanation. Despite its low metallicity and the concomitant high electron temperature that should result in collisionally excited Balmer lines of $\mathrm{H}$, PNG $135.9+55.9$ has an $\mathrm{H} \alpha / \mathrm{H} \beta$ ratio typically below 3 . Furthermore, $\mathrm{H} \alpha / \mathrm{H} \beta$ appears to be variable between observing runs and even within a single night. One possible explanation for both the low $\mathrm{H} \alpha / \mathrm{H} \beta$ ratio and its variability is if PNG 135.9+55.9 contains an accretion disk, though the evidence is not convincing. At any rate, this issue does not appear to affect our conclusions regarding the chemical abundances.

We also measure a low $\mathrm{He} / \mathrm{H}$ ratio of $\sim 0.08$. This makes PNG 135.9+55.9 interesting as a probe of the pregalactic He abundance. However, the derivation of a very precise He abundance will require the resolution of a number of outstanding issues, including the $\mathrm{H} \alpha / \mathrm{H} \beta$ problem, the foreground reddening, and the internal temperature structure.

Acknowledgements. MGR thanks Anabel Arrieta, Leonid Georgiev, Felipe Montalvo, and Salvador Monrroy for their able assistance with the observations at SPM. GT, GS, MGR, and CV are grateful for the receipt of Director's discretionary time at the CFHT. We are grateful to the WHT staff for the spectrum obtained through service time. GS acknoledges useful discussions with Y. Izotov, J. M. Huré and M. Mouchet. PDD is a PPARC-supported PDRA. MGR acknowledges financial support from DGAPA project IN100799 and CONACyT project 37214-E. GS acknowledges financial support from DGAPA project IN114601. GT and GS acknowledge financial support from CONACyT project 34521-E. 


\section{References}

Allende Prieto, C., Lambert, D. L., \& Asplund, M. 2001, ApJ, 556, L63

Aller, L. H. 1987, Physics of Thermal Gaseous Nebulae (Dordrecht, The Netherlands: D. Reidel Publishing Company)

Barlow, M. J. 1987, MNRAS, 227, 161

Bedding, T. R., \& Zijlstra, A. A. 1994, A\&A, 283, 955

Benjalin, R. A., Skillman, E. D., \& Smits, D. P. 1999, ApJ, 514, 307

Blöcker, T. 1995, A\&A, 299, 755

Boesgaard, A. M., King, J. R., Deliyannis, C. P., \& Vogt, S. S. 1999, AJ, 117, 492

Burris, D. L., Pilachowski, C. A., Armandroff, T. E., et al. 2000, ApJ, 544,302

Corradi, R. L. M. 1995, MNRAS, 276, 521

Esteban, C., Peimbert, M., Torres-Peimbert, S., \& Escalante, V. 1998, MNRAS, 295, 401

Ferland, G. J., Korista, K. T., Verner, D. A., et al. 1998, PASP, 110, 761

Filippenko, A. V. 1982, PASP, 94, 715

Fitzpatrick, E. L. 1999, PASP, 111, 63

Grevesse, N., \& Sauval, A. J. 1998, Space Sci. Rev., 85, 161

Henry, R. B. C. 1989, MNRAS, 241, 453

Howard, J. W., Henry, R. B. C., \& McCartney, S. 1997, MNRAS, 284, 465

Israelian, G., Rebolo, R., García López, R. J., et al. 2001, ApJ, 551, 833

Kingsburgh, R. L., \& Barlow, M. J. 1994, MNRAS, 271, 257
Lee, H.-W., \& Yhung, S. 2000, ApJ, 530, L49

Le Fèvre, O., Crampton, D., Felenbok, P., \& Monnet, G. 1994, A\&A, 282,325

Livio, M., \& Soker, N. 2001, ApJ, 552, 685

Lucy 1974, AJ, 79, 745

McCall, M. L., \& Armour, M. H. 2000, in Mapping the Hidden Universe: The Universe Behind the Milky Way - The Universe in Hi, ASP Conf. Ser., 218, ed. R. C. Kraan-Korteweg, P. A. Henning, \& H. Andernach (San Francisco: Astronomical Society of the Pacific)

McCall, M. L., Rybski, P. M., \& Schields, G. A. 1985, ApJS, 57, 1

McGaugh, S. S. 1991, ApJ, 380, 140

Miranda, L. F., \& Torrelles, J. M. 1998, ApJ, 496, 274

Oke, J. B. 1990, AJ, 99, 1621

Rauch, T. 2002, Rev. Mex. Astron. Astrofis. (Serie de Conferencias), 12,150

Richardson 1972, J. Opt. Soc. Am., 62, 55

Schlegel, D. J., Finkbeiner, D. P., \& Davis, M. 1998, ApJ, 500, 525

Stasińska, G., \& Leitherer, C. 1996, ApJS, 107, 661

Storey, P. J., \& Hummer, D. G. 1995, MNRAS, 272, 41

Thielemann, F., Nomoto, K., \& Hashimoto, M. 1996, ApJ, 460, 408

Tovmassian, G. H., Stasińska, G., Chavushyan, V. H., et al. 2001, A\&A, 370, 456

van Hoof, P. A. M. 2000, MNRAS, 314, 99

Warner, B. 1995, Cataclysmic Variable Stars (Cambridge Univ. Press, Cambridge)

Williams, G. A. 1995, AJ, 109, 319

Woosley, S. E., \& Weaver, T. A. 1995, ApJS, 101, 181 